\title{
AVALIAÇÃO DA ATENÇÃO EM DIABETES MELLITUS EM UMA UNIDADE BÁSICA DISTRITAL DE SAÚDE
}

\author{
Adriana Serafim Bispo e Silva1, Manoel Antônio dos Santos², Carla Regina de Souza Teixeira ${ }^{3}$, Marta Maria \\ Coelho Damasceno ${ }^{4}$, Josana Camilo ${ }^{5}$, Maria Lúcia Zanetti ${ }^{6}$
}

\footnotetext{
${ }^{1}$ Doutora em Enfermagem. Enfermeira da Secretaria Municipal de Saúde de Ribeirão Preto - SP. São Paulo, Brasil E-mail: madribispo@uol.com.br

${ }^{2}$ Doutor em Psicologia. Professor da Faculdade de Filosofia, Ciências e Letras da Universidade de São Paulo (USP). São Paulo, Brasil E-mail: masantos@ffclrp.usp.br

${ }^{3}$ Doutora em Enfermagem. Professor Doutor do Departamento de Enfermagem Geral e Especializada. Escola de Enfermagem de Ribeirão Preto (EERP) da USP. São Paulo, Brasil E-mail: carlarst@eerp.usp.br

${ }^{4}$ Doutora em Enfermagem. Professor do Departamento de Enfermagem. Universidade Federal do Ceará. São Paulo, Brasil E-mail: martadamasceno@terra.com.br

${ }^{5}$ Aluna do curso de Licenciatura e Bacharelado em Enfermagem da EERP-USP. Bolsista CNPq. São Paulo, Brasil E-mail: josana. camilo@usp.br

${ }^{6}$ Doutora em Enfermagem. Professor Associado do Departamento de Enfermagem Geral e Especializada da EERP-USP. São Paulo, Brasil E-mail: zanetti@eerp.usp.br
}

RESUMO: Estudo quantitativo transversal com objetivo de avaliar os componentes estrutura e processo na atenção ao usuário com Diabetes Mellitus em uma Unidade Básica Distrital de Saúde do município de Ribeirão Preto-SP. Foram analisados 138 prontuários de usuários com Diabetes Mellitus, em 2008. Como referencial metodológico, utilizou-se a Avaliação de Cuidados em Saúde tendo como recorte a estrutura e o processo. Um formulário contendo 24 questões fechadas foi empregado para a coleta de dados. Em relação à estrutura, os resultados mostraram que os recursos físicos e materiais eram adequados. Quanto ao processo, as ações implementadas na assistência aos usuários com Diabetes Mellitus encontravam-se fragmentadas. Conclui-se que é preciso repensar o modo de organização do trabalho, tornando as ações de saúde comprometidas com uma prática equitativa de qualidade, que promova a comunicação entre os diversos profissionais envolvidos na atenção em Diabetes e o cumprimento pleno de suas atribuições e competências.

DESCRITORES: Avaliação em saúde. Diabetes mellitus. Enfermagem. Recursos humanos.

\section{EVALUATING DIABETES MELLITUS CARE IN A BRAZILIAN BASIC HEALTH DISTRICT}

\begin{abstract}
The objective of this cross-sectional quantitative study is to evaluate the structure and process components in Diabetes Mellitus user care given in a Basic Health District of Ribeirão Preto, SP, Brazil. We analyzed 138 medical records from users with Diabetes Mellitus in 2008. As a methodological reference, we used the Health Care Assessment using structure and process as the cut. A questionnaire containing 24 closed questions was used to collect data. Concerning the structure, the results showed that physical and material resources were adequate. As for the process, the actions implemented in assisting users with Diabetes Mellitus were fragmented. It follows that we must rethink the way such work is organized, helping health actions become committed to a fair quality practice, which promotes communication among the various professionals involved in Diabetes care and full compliance with their duties and responsibilities

DESCRIPTORS: Health evaluation. Diabetes mellitus. Nursing. Human resources.
\end{abstract}

\section{EVALUACIÓN DE LA ATENCIÓN A PERSONAS CON DIABETES MELLITUS EN UNA UNIDAD BÁSICA DE SALUD}

\begin{abstract}
RESUMEN: Estudio cuantitativo de corte transversal con el objetivo de evaluar los componentes, estructura y proceso en la atención a personas con Diabetes Mellitus en una Unidad Básica de Salud de la ciudad de Ribeirão Preto-SP. Se analizaron 138 historias clínicas de personas con Diabetes Mellitus, en 2008. Como metodología se utilizó la Evaluación de la Atención de la Salud, y como recorte, la estructura y el proceso. Para la colecta de datos se utilizó un cuestionario con 24 preguntas cerradas. Los resultados mostraron que los recursos físicos y materiales eran adecuados. Las acciones realizadas en la atención a personas con Diabetes se encontraban fragmentadas. Se concluye que es necesario repensar la forma de organizar el trabajo, para que las acciones de salud sean comprometidas con una práctica equitativa de calidad, que promueva la comunicación entre los profesionales implicados en la atención a personas con Diabetes y el cumplimiento pleno de sus deberes y responsabilidades.
\end{abstract}

DESCRIPTORES: Evaluación en salud. Diabetes mellitus. Enfermería. Recursos humanos. 


\section{INTRODUÇÃO}

O Diabetes Mellitus (DM) é uma doença crônica de alta prevalência no Brasil e é considerado um problema de saúde pública. ${ }^{1-2}$ Estudo realizado em Ribeirão Preto-SP mostrou que a prevalência de DM na população de 30 a 69 anos é de 12,1\%. ${ }^{3}$ Esse dado aponta para a magnitude desse agravo no município, o que evidencia a necessidade de reorganização da atenção aos usuários acometidos.

A atenção em DM direciona-se para a oferta de intervenções clínicas e educativas baseadas em evidências científicas, que deverão compor o plano de tratamento e contribuir para o alcance de resultados satisfatórios, principalmente em relação ao controle metabólico. ${ }^{4}$ Nesse contexto, destaca-se a elaboração de protocolos como recurso importante para o planejamento sistemático e implementação da assistência aos usuários com DM. ${ }^{5-6}$ Protocolos internacionais, nacionais e municipais têm proposto que a assistência em DM seja conduzida por uma equipe multiprofissional na intenção de promover cuidado integral, equitativo, resolutivo e de qualidade., ${ }^{4,-8}$

Desse modo, o Protocolo de Atendimento em Hipertensão Arterial e Diabetes Mellitus, elaborado pela Secretaria Municipal de Saúde de Ribeirão Preto-SP, utilizou esses princípios como fundamento, com vistas a melhorar a qualidade de vida dos usuários e, no âmbito da gestão, reduzir gastos com hospitalizações e procedimentos de alta tecnologia. ${ }^{8}$

Entende-se que os protocolos, como norteadores da assistência integral ao usuário, devem ser alvo de avaliações frequentes, em particular no que diz respeito ao seguimento das suas recomendações. No entanto, o Protocolo mencionado anteriormente vem sendo implementado no município desde 2006 até o presente momento, sem contar com uma avaliação para evidenciar os benefícios e as limitações desse recurso.

\section{Quadro teórico}

A avaliação de serviços de saúde é definida como um instrumento de gestão e planejamento baseado em um julgamento explícito e dinâmico que, ao seu fim, desencadeia um movimento de transformação nas práticas e serviços de saúde, na direção de uma qualidade previamente almejada. ${ }^{9-10}$ Para tanto, pode ser delimitada a partir da análise dos recursos humanos, dos recursos materiais e do cuidado dispensado ao usuário. ${ }^{10}$ No quadro conceitual de avaliação de cuidado em saúde apresentado por Avedis Donabedian encontra-se que a avaliação deve ser pautada em três componentes: a estrutura, o processo e o resultado. ${ }^{10}$ Esse referencial teórico propõe uma avaliação sistêmica da qualidade da atenção em saúde e é amplamente utilizado como base para realização de pesquisas de avaliação de serviços, programas ou tecnologias em saúde. ${ }^{10}$

A estrutura refere-se, fundamentalmente, às características dos insumos empregados na atenção em saúde, que compreendem tanto as informações sobre recursos físicos, humanos ou materiais, como as formas de organização e funcionamento (normas e rotinas) que regulam a oferta do cuidado em saúde. No processo, analisa-se a competência da equipe de saúde no manejo do processo saúde-doença, a partir da adequação das ações ao conhecimento técnico e científico vigente. $\mathrm{O}$ resultado abrange os efeitos que as ações e procedimentos executados provocam na clientela assistida. ${ }^{10}$

Para este estudo, elegeu-se a avaliação de um aspecto do componente estrutura, ou seja, os recursos humanos que compõem a equipe de saúde envolvida na assistência aos usuários com DM. No que diz respeito ao componente processo, considerou-se o número e o tipo de atividades assistenciais realizadas pelos profissionais, bem como o tipo e número de exames laboratoriais registrados no prontuário dos usuários com DM.

Nesse contexto, destaca-se a relevância do prontuário de saúde como um documento estratégico para avaliar a assistência prestada ao usuário. Os registros ali contidos são utilizados para a tomada de decisão clínica, para fins de gestão, planejamento e apoio à pesquisa, configurando-se como elementos importantes para a obtenção de informações acerca do processo de trabalho em saúde. ${ }^{11}$ No processo de reorganização da atenção ao usuário com DM, os profissionais de saúde da Unidade sob investigação no presente estudo enfrentam dificuldades relacionadas aos recursos físicos e materiais, composição da equipe mínima necessária para o atendimento aos usuários com DM e à operacionalização das ações em saúde. Desse modo, a realização de um estudo de avaliação mostra-se relevante para obtenção de subsídios que possam dar suporte ao processo de reorganização da atenção ao usuário com DM na referida Unidade. Entende-se que essa avaliação pode favorecer a compreensão dos problemas relacionados à estrutura e ao processo da assistência, que podem comprometer a atenção em DM.

Pelo exposto, este estudo teve como objetivo avaliar componentes da estrutura e o processo da atenção ao usuário com DM em uma 
Unidade Básica Distrital de Saúde do município de Ribeirão Preto-SP.

\section{MÉTODO}

Trata-se de um estudo quantitativo transversal, realizado em uma Unidade Básica Distrital de Saúde de Ribeirão Preto-SP, organizada para a oferta de atenção básica e de média complexidade. Os dados foram obtidos no período de julho a dezembro de 2008. Inicialmente, foram selecionados 333 prontuários de saúde de usuários com DM elencados no Sistema de Cadastramento e Acompanhamento de Pacientes Hipertensos e Diabéticos da referida Unidade. Estabeleceu-se como critérios de inclusão os prontuários que tivessem registro do diagnóstico de $\mathrm{DM}$, além de dois ou mais registros de atendimentos médicos ou de enfermagem no período de janeiro de 2007 a julho de 2008. A escolha desse período justifica-se, uma vez que a Secretaria Municipal de Saúde de Ribeirão Preto implantou o Protocolo de Atendimento em Hipertensão e Diabetes em julho de 2006. Foram investigados 138 prontuários de saúde que atenderam aos critérios de inclusão. Cabe destacar que a Unidade de estudo tem média mensal de 22 mil atendimentos em saúde, porém não há dados sobre o número específico de atendimentos a usuários com DM.

No presente estudo, o componente estrutura referiu-se às categorias profissionais que compõem a equipe de saúde envolvida na assistência ao usuário com DM. No que tange ao processo, considerou-se o número de atendimentos em saúde realizado por categoria profissional e as atividades técnicas desempenhadas e registradas nos prontuários de saúde por ocasião da última consulta ou atendimento: pressão arterial, peso corporal e circunferência abdominal. Também foram incluídos os exames laboratoriais realizados cujos resultados foram registrados nos prontuários de saúde por ocasião da última consulta ou atendimento, a saber: hemoglobina glicada, glicemia de jejum, glicemia pós-prandial, colesterol total, fração de colesterol Low Density Lipoprotein (LDL), fração de colesterol High Density Lipoprotein (HDL), triglicerídeos, creatinina, uréia, proteinúria e microalbuminúria. Elegeram-se, ainda, as variáveis: registro unitário ou múltiplo da avaliação dos pés e da avaliação oftalmológica, bem como o registro da implementação de programa ou plano de estratégias educativas para promoção do autocuidado em DM.

Os dados foram obtidos pelos pesquisadores por meio de consulta aos prontuários dos usuários, realizada em uma sala reservada nas dependências da Unidade de Saúde. Essa opção está alicerçada na compreensão de que o registro em prontuário é elemento imprescindível no processo de cuidado em saúde, considerando seu potencial para documentar o delineamento e a implementação da assistência prestada, de modo a possibilitar a comunicação permanente e a continuidade do cuidado entre os membros da equipe de saúde segundo habilidades e competências específicas necessárias para gerir o cuidado em saúde.,12-13.

Utilizou-se como instrumento um formulário estruturado contendo 24 questões fechadas relacionadas às variáveis de estrutura (recursos humanos) e ao componente processo (o registro pelos profissionais de saúde dos parâmetros relacionados ao peso corporal, pressão arterial, Índice de Massa Corporal (IMC), Circunferência Abdominal (CA), avaliação dos pés e oftalmológica e exames laboratoriais). A coleta de dados teve duração média de, aproximadamente, 30 minutos por prontuário. A análise dos dados foi realizada com base na estatística descritiva. O Comitê de Ética em Pesquisa da Escola de Enfermagem de Ribeirão Preto da Universidade de São Paulo aprovou o projeto sob o Protocolo n. 0932/2008.

\section{RESULTADOS}

Quanto ao componente estrutura, particularmente no que diz respeito aos recursos humanos, os profissionais de saúde envolvidos no atendimento do usuário com DM foram identificados nos registros dos prontuários como: cardiologistas, endocrinologistas, enfermeiros, auxiliares de enfermagem e farmacêutico.

No que se refere ao componente processo obteve-se que os prontuários analisados apresentavam registros predominantemente de atendimentos realizados por médicos e auxiliares de enfermagem (Tabela 1).

Tabela 1 - Distribuição numérica e percentual de categorias profissionais que compõem a equipe de saúde envolvida na assistência ao usuário com Diabetes Mellitus. Ribeirão Preto-SP, 2008

\begin{tabular}{lrr}
\hline \multicolumn{1}{c}{ Profissional $^{*}$} & $\mathbf{n}$ & \multicolumn{1}{c}{$\%$} \\
\hline Médico & 138 & 100,0 \\
Auxiliar de enfermagem & 132 & 95,7 \\
Enfermeiro & 13 & 9,4 \\
Farmacêutico & 12 & 8,6 \\
Outros profissionais de saúde & 29 & 21,1 \\
\hline
\end{tabular}

* As categorias não são mutuamente excludentes. 
Os dados obtidos em relação às atividades técnicas realizadas pelos profissionais de saúde evidenciaram que os valores da pressão arterial estavam registrados na totalidade dos prontuários consultados. Vale ressaltar que não foram encontrados registros do IMC (Tabela 2).

Tabela 2 - Distribuição numérica e percentual de prontuários de saúde de usuários com Diabetes Mellitus segundo os registros de parâmetros utilizados para avaliação clínica: peso, pressão arterial, índice de massa corporal, circunferência abdominal. Ribeirão Preto-SP, 2008

\begin{tabular}{lrr}
\hline Parâmetros de avaliação clínica* & $\mathbf{n}$ & \% \\
\hline Níveis de pressão arterial & 138 & 100,0 \\
Peso corporal & 136 & 98,6 \\
Circunferência abdominal & 22 & 15,9 \\
IMC & - & - \\
\hline * As categorias não são muluamente
\end{tabular}

A avaliação dos pés e a avaliação oftalmológica foram registradas em apenas $21(15,2 \%)$ e seis $(4,3 \%)$ prontuários de saúde, respectivamente. A tabela 3 mostra o registro de resultados de exames laboratoriais no prontuário de saúde dos usuários com DM.

Tabela 3 - Distribuição numérica e percentual de prontuários de usuários com Diabetes Mellitus com registro de resultados de exames laboratoriais. Ribeirão Preto-SP, 2008

\begin{tabular}{lrr}
\hline Exames laboratoriais & $\mathbf{n}$ & $\mathbf{\%}$ \\
\hline Glicose de jejum & 129 & 93,5 \\
Hemoglobina glicada & 112 & 81,2 \\
Glicose pós-prandial & 89 & 64,5 \\
Colesterol total & 122 & 88,4 \\
Colesterol LDL & 122 & 88,4 \\
Colesterol HDL & 120 & 87,0 \\
Triglicérides & 121 & 87,7 \\
Creatinina & 116 & 84,1 \\
Uréia & 71 & 51,4 \\
Proteinúria & 45 & 32,6 \\
Microalbuminúria & 2 & 1,4 \\
\hline
\end{tabular}

Em relação à implementação de programa ou plano de estratégias educativas para promoção do autocuidado em DM, verificou-se falta de registro sistemático acerca dos mesmos. Desse modo, não há estratégias individuais ou grupais registradas nos prontuários. Os dados mostram que os registros nos prontuários restringiam-se às orientações quanto à alimentação e prescrição do tratamento não medicamentoso. Esses registros eram pouco detalhados e se referiam apenas à redução de peso, sem a abordagem dos grupos alimentares, número de refeições, intervalos e restrições, bem como sua relação com os medicamentos prescritos. Quanto à atividade física, os registros referiam à prescrição dessa atividade, porém sem anotações acerca dos benefícios e aspectos relacionados ao tipo de atividade, horário, cuidado com a alimentação, tempo de realização, entre outros.

\section{DISCUSSÃO}

Os dados referentes às categorias profissionais que compõem a equipe de saúde envolvida na assistência ao usuário com DM mostraram que a maior frequência de registro foi realizada pelos profissionais médicos e auxiliares de enfermagem, e a menor frequiência, pelos enfermeiros e os farmacêuticos. O Protocolo de Atendimento em Hipertensão e Diabetes recomenda que os profissionais médico, enfermeiro, auxiliar de enfermagem, farmacêutico, odontólogo e assistente social devem compor a equipe mínima necessária para assistência em DM nas Unidades Básicas de Saúde. ${ }^{8}$ A frequência elevada de registros de atendimento dos profissionais médicos e auxiliares de enfermagem aponta que o modelo de organização do trabalho está centrado no modelo biomédico.

De acordo com o referido Protocolo, os auxiliares de enfermagem, na assistência ao usuário com DM, devem desempenhar atividades relacionadas aos procedimentos técnicos de enfermagem, tais como verificação do peso corporal, aferição da pressão arterial, glicemia capilar, entre outros, o que pode justificar a elevada frequência de registros pelos referidos profissionais. No entanto, o auxiliar de enfermagem deve contribuir na realização de procedimentos técnicos no contexto das intervenções de enfermagem, com vistas ao alcance de resultados efetivos da atenção, e não somente na realização de procedimentos complementares ao trabalho em saúde. ${ }^{14}$ Se por um lado, preconiza-se a relação horizontal da equipe de saúde, que permitiria a interface das ações mantendo-se as atribuições e competências específicas de cada profissional, por outro lado, os dados mostraram que o enfermeiro tem documentado pouco a sua prática profissional realizada com usuários com $\mathrm{DM}$, em contraste com o que reza a legislação profissional. $^{15}$

A escassez de registro realizada pelos enfermeiros implica em prejuízo no processo de cuidado 
de pessoas com DM, especialmente no que se refere à análise da qualidade da assistência prestada, visando ao alcance da integralidade em saúde.

Por outro lado, a deficiência de registros realizados por enfermeiros, farmacêuticos e outros profissionais de saúde, em número significativo, evidencia a fragmentação do processo de trabalho em saúde, comprometendo o cuidado inovador em diabetes. A diferença na frequência dos registros por categoria profissional reforça a compreensão de que os profissionais, na implementação de suas ações, ainda não conseguem agregar elementos que os possam configurar como uma equipe multiprofissional para o cuidado. ${ }^{16}$

Os dados sugerem que, nas ações do farmacêutico e do enfermeiro, como membros efetivos da equipe de saúde envolvida na assistência em DM, estão sendo subtraídas habilidades e atribuições específicas destes profissionais de saúde. $\mathrm{O}$ que pode levar a um empobrecimento da atuação desses profissionais, do ponto de vista das ações registradas no prontuário. Nessa vertente, outros estudos são necessários para identificar o modo de organização do trabalho em saúde para seguimento de usuários com DM, incluindo ações além daquelas documentadas em prontuário, mas que possam configurar-se em trabalho vivo na atenção em DM.

Em relação ao componente processo, no que tange aos registros de parâmetros para avaliação clínica: peso, pressão arterial, índice de massa corporal, circunferência abdominal, verificou-se que a operacionalização de ações e procedimentos técnicos, tais como aferição da pressão arterial e do peso corporal, estava registrada no prontuário de saúde para a maioria dos atendimentos aos usuários com DM. Por outro lado, os registros do IMC e da circunferência abdominal ainda não constituem componentes da avaliação dos usuários na Unidade investigada.

O cuidado integral à pessoa com DM aponta que a avaliação de saúde deve contemplar a mensuração de parâmetros que permitam a composição da análise clínica de risco de doenças cardiovasculares e de levantamento de necessidades para a implementação do cuidado de enfermagem, de modo a alcançar os objetivos de um plano assistencial comum. ${ }^{4,8}$

O IMC, a circunferência abdominal e a verificação da pressão arterial são considerados parâmetros simples e de baixo custo para avaliação clínica do usuário com DM. Esses parâmetros podem ser obtidos pelo médico e auxiliar de enfermagem, conforme já mencionado. Por outro lado, espera-se que o enfermeiro seja responsável pelo treinamento e supervisão dos auxiliares de enfermagem na realização desses procedimentos, conforme proposto no Protocolo de Atendimento em Hipertensão e Diabetes. ${ }^{8,17}$ Como responsável pelo treinamento e supervisão, era esperada maior frequência de registros desses parâmetros pelo enfermeiro.

Os dados mostram que o papel gerencial do enfermeiro, no que se refere ao planejamento, coordenação, supervisão e treinamento para oferta de assistência do usuário com DM, está aquém daqueles definidos pelo Protocolo. Nessa vertente, aponta-se a importância do enfermeiro assumir suas atribuições quanto ao treinamento e supervisão da equipe de enfermagem, bem como a coordenação das ações que garantam a verificação e registro dos parâmetros clínicos de risco cardiovascular, a fim de alcançar os indicadores de qualidade da assistência de enfermagem aos usuários com DM.

No que se refere à avaliação dos pés, verificou-se que a frequência de registros nos prontuários é baixa. Esse dado mostra que o exame dos pés e as condutas clínicas e educativas associadas a esse procedimento ainda não se constituem em informações disponíveis para o planejamento, implementação e avaliação de ações para o autocuidado com os pés. Nesse contexto, o usuário pode estar em risco, uma vez que foram observadas lacunas nos prontuários em relação à identificação de fatores preditivos de risco para úlceras e amputações, bem como em relação ao registro de medidas preventivas, curativas e educativas para o cuidado com os pés. ${ }^{8}$

Quanto à avaliação oftalmológica, verificou-se a falta de registros sistemáticos acerca da solicitação, realização e resultados de exames para rastreio e classificação da retinopatia diabética. Esse dado mostra que os profissionais não estão seguindo as recomendações do Protocolo para avaliação oftalmológica, que preconiza que a mesma deve ser realizada anualmente, sendo a partir do quinto ano para os usuários com DM tipo 1 e a partir do diagnóstico para DM tipo 2, ou não estão registrando e arquivando os exames solicitados e seus respectivos resultados. Nessa direção, ações relacionadas à qualidade do registro necessitam ser revisadas no referido local de estudo, a fim de imprimir maior qualidade ao processo de trabalho na atenção em DM. Os resultados da avaliação oftalmológica, assim como outros resultados de exames de rastreamento e diagnóstico, são im- 
prescindíveis para subsidiar a implementação de intervenções de enfermagem relacionadas às ações de autocuidado aos usuários com comprometimento visual, tais como aplicação de insulina, cuidado com os pés, entre outras. ${ }^{17}$

No que tange à avaliação laboratorial, verificou-se que a maioria dos exames registrados refere-se ao perfil glicêmico, seguido do lipídico. Porém, a hemoglobina glicada não foi registrada como exame de excelência para avaliação do perfil glicêmico e acompanhamento da terapêutica medicamentosa prescrita. ${ }^{4,8}$

Quanto à avaliação do perfil renal, verificou-se que o registro com maior frequência foi dos valores de creatinina, seguidos de uréia. No entanto, a análise dessa variável ficou comprometida, pois não foi verificado o registro dos valores de proteinúria para os usuários com DM da referida Unidade, uma vez que o Protocolo recomenda a solicitação do exame de microalbuminúria somente para aqueles usuários que apresentam proteínas no exame de urina rotina ${ }^{8}$.

Os resultados encontrados no presente estudo estão em concordância com uma pesquisa realizada no estado do Rio Grande do Sul. ${ }^{18}$ Esse estudo mostrou que os responsáveis pelo acompanhamento ambulatorial do usuário com DM também não solicitaram, para a totalidade dos usuários investigados, os exames laboratoriais mínimos, tais como glicemia de jejum, hemoglobina glicada e perfil lipídico.

$\mathrm{Na}$ consulta de enfermagem, o enfermeiro deve solicitar os exames laboratoriais para subsidiar a avaliação clínica de saúde e estratificação de pessoas com DM segundo critérios de adesão ao tratamento. ${ }^{8}$ Assim, aponta-se a necessidade do enfermeiro assumir seu papel assistencial e gerencial no cuidado à pessoa com DM, contribuindo para a qualificação do cuidado e alcance dos princípios de integralidade da assistência.

Quanto à implementação de programa e/ou plano de estratégias educativas para promoção do autocuidado em DM, os dados mostraram que há escassez de registro acerca das ações educativas sistematizadas. O processo educativo em diabetes, parte integrante do atendimento clínico, é imprescindível para o alcance do controle metabólico do usuário com DM. ${ }^{4,6,8,19} \mathrm{O}$ enfermeiro tem papel relevante na condução e seguimento educativo dos usuários. A falta de ações educativas sistematizadas visando ao automanejo da doença aponta para a fragmentação do processo assistencial, o que pode comprometer a adesão dos usuários ao tratamento preconizado, aumentando os custos de atendimento à saúde. ${ }^{6,20-21}$

\section{CONCLUSÃO}

Os resultados deste estudo mostraram que, em relação à estrutura, particularmente no que concerne aos recursos humanos, a Unidade de Saúde mostra-se em conformidade com a equipe preconizada no Protocolo de Atendimento em Hipertensão e Diabetes.

Em relação ao processo, os dados evidenciaram que a assistência ao usuário com DM encontra-se fragmentada. Os profissionais que compõem a equipe de saúde trabalham segundo o modelo biomédico, sendo que os registros das ações em saúde foram predominantemente realizados pelos médicos e auxiliares de enfermagem. Dentre as ações preconizadas no Protocolo, constatou-se que o exame dos pés, o IMC, a CA, o exame oftalmológico, a hemoglobina glicada e as ações educativas ainda não foram implementadas na sua totalidade. Por outro lado, ao considerar as ações registradas no prontuário, estas mostraram que há falta de integração e articulação do processo de trabalho.

Os resultados apontam a necessidade de reconhecer a avaliação como um processo permanente para o alcance da qualidade na atenção em DM. Nessa direção, é preciso elaborar um plano assistencial comum, visando à identificação, elaboração de diagnósticos de saúde, implementação e avaliação de intervenções aos usuários, alicerçado em um processo gerencial no qual os protocolos descritivos possam ser transformados em intervenções concretas, no contexto do serviço de saúde.

Espera-se que o enfermeiro, como elemento articulador do processo de trabalho, ajude a repensar a forma de organização multidisciplinar, comprometido com uma prática equitativa de qualidade, que promova a comunicação entre os diversos profissionais de saúde envolvidos na atenção em Diabetes, o cumprimento de suas atribuições e competências de maneira plena, que possam ser corroboradas pelos registros em prontuários de saúde dos usuários.

Neste estudo foram avaliadas algumas características dos componentes estrutura e processo. Frente ao volume e complexidade dos componentes referentes aos resultados, estes serão apresentados e analisados em outra publicação. No presente estudo, temos como limitação a coleta dos dados em prontuário em detrimento de 
entrevistas aos profissionais de saúde envolvidos na atenção em Diabetes para apreender o componente processo na atenção em Diabetes. Propõe-se que sejam realizados estudos futuros que dêem voz aos profissionais de saúde e usuários para o aprofundamento do fenômeno de avaliação de serviços de atenção à pessoa com $\mathrm{DM}$, com vistas à comparação de resultados e ao alcance da qualidade do atendimento.

\section{REFERÊNCIAS}

1. Wild SH, Roglic G, Green A, Sicree R, King H. Global prevalence of diabetes estimates for the year 2000 and projections for 2030. Diabetes Care. 2004 May; 27(5):1047-53.

2. Malerbi DA, Franco LJ. Multicentric study of the prevalence of diabetes melito and impaired tolerance in urban Brazilian population aged 30-69 yr. Diabetes Care 1992 Nov; 15(11):1509-16.

3. Torquato MT, Montenegro Júnior RM, Viana LA, Souza RA, Lanna CM, Lucas JC, et al. Prevalence of diabetes mellitus and impaired glucose tolerance in the urban population aged 30-69 years in Ribeirão Preto (São Paulo), Brazil. São Paulo Med J. 2003; 121(6):224-30.

4. American Diabetes Association (ADA). Standards of medical care in diabetes. Diabetes Care. 2009; 32(Suppl 1):S13-57.

5. Sociedade Brasileira de Diabetes (SBD). Diretrizes da Sociedade Brasileira de Diabetes. São Paulo (SP): Sociedade Brasileira de Diabetes; 2008.

6. Funnell M, Haas L. National standards for diabetes self-management education programs. Diabetes Care. 1995 Jan; 18(1):100-16

7. Ministério da Saúde (BR). Diabetes mellitus. Brasília. (DF): Ministério da Saúde; 2006.

8. Secretaria Municipal da Saúde (SP). Protocolo de atendimento em hipertensão e diabetes. Ribeirão Preto (SP): Prefeitura Municipal. Secretaria Municipal da Saúde; 2006.

9. Tanaka OY, Melo C. Avaliação de programas de saúde do adolescente: um modo de fazer. São Paulo (SP): Edusp; 2001.

10. Donabedian A. Basic approaches to assessment: structure, process and outcome. In: Donabedian A.
Explorations in quality assessment and monitoring. Michigan (US): Health Administration Press; 1980. p. 77-125.

11. Donabedian A. The quality of care: how can it be assessed? JAMA. 1988 Sep; 260(12):1743-8.

12. Matsuda LM, Silva DMPP, Évora YM, Coimbra, JAH. Anotações/registros de enfermagem: instrumento de comunicação para a qualidade do cuidado? Rev Eletrônica Enferm. 2006; 8(3):415-21.

13. Organização Mundial de Saúde. Cuidados inovadores para condições crônicas: componentes estruturais de ação: relatório mundial. Brasília (DF): Organização Mundial de Saúde; 2003.

14. Conselho Federal de Enfermagem (COFEN). Resolução COFEN n ${ }^{\circ}$ 358/2009 de15 de novembro de 2009. Dispõe sobre a Sistematização da Assistência de Enfermagem e a implementação do processo de enfermagem em ambientes públicos ou privados, em que ocorre o cuidado profissional de Enfermagem e dá outras providências. 2009. Diário Oficial da União (BR): 203; secção 1; 179.

15. Cruz, DALM. Processo de enfermagem e classificações. In: Gaidzinski RR, Soares, AVN, Costa Lima AF, Gutierrez BAO, Cruz DALM, Rogenski NMB, Sancinetti TR, organizadores. Diagnósticos de enfermagem na prática clínica. Rio de Janeiro (RJ): Artmed; 2008. p. 25-37.

16. Peduzzi M. Equipe multiprofissional de saúde: conceito e tipologia. Rev Saúde Pública. 2001 Fev; 35(1):103-9.

17. Andrade NHS, Zanetti ML, Santos MA. A percepção visual de pacientes com retinopatia diabética segundo o referencial de Merleau-Ponty. Rev Enferm UERJ. 2008 Abr-Jun; 16(2):249-54.

18. Assunção MCF, Santos IS. Gigante DP. Atenção primária em diabetes no sul do Brasil: estrutura, processo e resultado. Rev Saúde Pública. 2001 Fev; 35(1):88-95.

19. Sociedade Brasileira de Diabetes (SBD). Diretrizes da Sociedade Brasileira de Diabetes. São Paulo (SP): SBD; 2009.

20. Xavier ATF, Bittar DB, Ataide MBC. Crenças no autocuidado em diabetes: implicações para a prática. Texto contexto - enferm. 2009 Jan-Mar; 18(1):124-30.

21. Gimenes HT, Zanetti ML, Haas VJ. Factors related to patient adherence to antidiabetic drug therapy. Rev Latino-am Enfermagem. 2009 Feb; 17(1):46-51. 